\title{
RRM Strategy Based on Throughput and Fairness in LTE-A Relay System
}

\author{
Jintao DU*, Jihong ZHAO** \\ *Xi'an University of Posts \& Telecommunications, China \\ **Nanjing University of Posts \& Telecommunications, China \\ kashi87@sina.cn, eeleeg@gmail.com
}

\begin{abstract}
Relay has been one of the most promising techniques towards LTE-A system, which improves cell coverage and throughput in cell edge. For the balance between system throughput and user fairness of LTE-A system with relay, this paper proposes user SINR dispersion degree as the main parameter for resource allocation, significantly improved the fairness of the center users and edge users. Simulation results show that the proposed Balanced Aim allocation algorithm which combined throughput, user fairness and game theory makes the system resource allocation more efficient, improving the overall performance of the system.
\end{abstract}

Key words - LTE-A; relay; game theory; throughput; user fairness

\section{INTRODUCTION}

LTE (Long Term Evolution) based on 3G technology, is the quasi-4G technology between $3 \mathrm{G}$ and $4 \mathrm{G}$ mobile communications. The LTE-Advanced(LTE-A) was formally proposed in 3GPP LTE Rel. 10 to expand, enhance and improve LTE technology, which is the core of LTE-A.

Currently LTE-A relay system is researched mainly about relay access selection [1-3] and the resource allocation problem in LTE-A system with relay [4], meanwhile relay cooperative communication has also been a growing number of considerations [5-7].Throughput maximization is the most intuitive system design goal in LTE-A Radio Resource Management (RRM), and one first studied issue of LTE-A relay network resource management. But this is the only point of view from the system, which does not consider the needs of users as well as the fairness factors among users. And this will result in being difficult to gain resources for users with relatively poor channel conditions. In the actual system design, the focus of researches about user fairness is to find a compromise or balance between maximizing system throughput and ensuring users' fairness.

There are several RRM realization options which have been discussed in the context of relay enhanced cellular networks in the literature. Scheduling algorithms for Relay Enhanced Cells (REC) can be centralized [8,9] where the evolved Node B (eNB) makes all scheduling decisions based on information about User Equipment (UE) buffers at Relay Nodes (RNs) and channel quality of the UEs. The algorithm achieves fair distribution of throughput at the BS of the same QoS class and load-balancing among different RNs and the BS in the cell. Such centralized algorithms can achieve near-optimal solution at the price of high signaling volume [9].

The main work for resource allocation in LTE-A relay system is based on the assumption that two-hop relay transmission occupying the same partition resources [10-12]. Although the literature [11] proposed adaptive time-domain resource partition proposal, but they only consider these programs to maximize throughput without taking the impact of fairness into account. Therefore, for the balance between cell throughput and user fairness in LTE-A relay system, this paper proposes user SINR dispersion degree as the main parameter for resource allocation, significantly improved the fairness of the center users and edge users. The balance allocation method which combined throughput, user fairness and game theory makes the system resource allocation more efficient.

The rest of this paper is organized as follows. In section 2, we outline the system model and definite the SINR dispersion 
degree. In section 3, we present the algorithm for the game between cell throughput and user fairness, and analyze the proposed scheme. The simulation results are analyzed in section 4 . The conclusion and future research are provided in section 5 .

\section{SYSTEM MODEL}

It is considered that the downlink two-hop communications are in the relay based architecture of a LTE-A network. In the cell of reference, there are a set of users (UEs) randomly distributed and two kind of serving nodes: one eNB and several RNs. RNs and eNB are referred as radio access points (RAP). Links in REC are divided into three classes: direct (eNB-UE), access (RN-UE) and backhaul (eNB-RN) [13]. The eNB serves $k$ UEs (Center UE) and the RNs serve $l$ UEs (Edge UE) as shown in Figure 1.

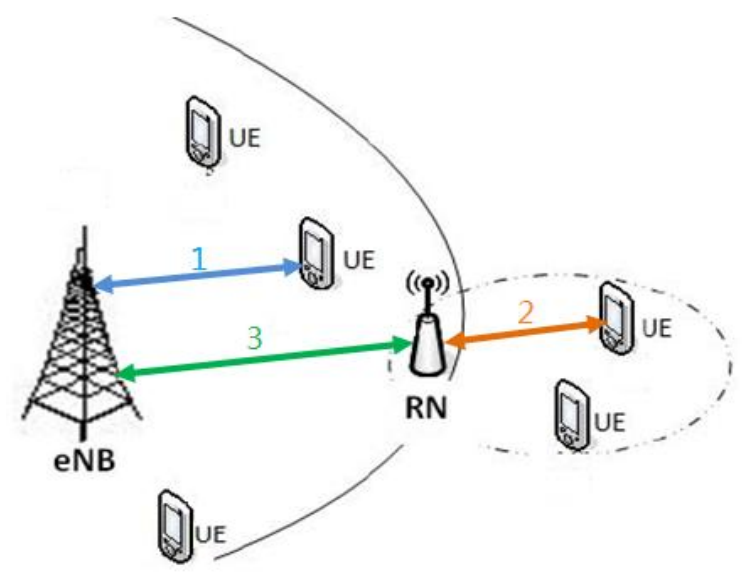

Figure 1. System model

Considering the signal during transmission attenuation factor of all and the impact of noise and interference, we determine that the user accesses eNB or RN by the size of the user's SINR values .In order to distinguish directly connected users and relay users, the algorithm is described as follows:

1) Select the RN which is the nearest RN for the user from all the RNs in the cell.

2) Calculate the user's SINR from eNB and the selected RN to the user, and choose the node with the larger SINR as the final node to communicate with the user.

$$
\text { node }=\max \left\{\operatorname{SINR}_{\mathrm{R}}, \operatorname{SINR}_{\mathrm{BS}}\right\}
$$

Where $\operatorname{SINR}_{R}$ and $\operatorname{SINR}_{\mathrm{BS}}$ are the SINR values from RN and eNB.

According to the idea of proportional fairness, the fairness of the user and the user's channel conditions are directly related. We propose the concept of User SINR Dispersion (USD), which reflecting user channel quality of the overall situation and the relative distribution more intuitively.

User SINR Dispersion: It is assumed that there are $M$ users in a micro-cell, the maximum SINR is defined as $S$, the $S$ were divided into $L$ segments, the method of calculating the User SINR Dispersion is defined as follows:

$$
\begin{aligned}
& \mathrm{D}_{\mathrm{j}}=\frac{1}{\mathrm{~L}} \sum_{\mathrm{m}=1}^{\mathrm{M}}\left(\sum_{\mathrm{i}=1}^{\mathrm{L}} \mathrm{D}\left(\mathrm{C}_{\mathrm{m}, \mathrm{i}}\right) \cdot \mathrm{i}-\mathrm{d}\right)^{2} \\
& \mathrm{~d}=\frac{1}{\mathrm{M}} \sum_{\mathrm{m}=1}^{\mathrm{M}} \sum_{\mathrm{i}=1}^{\mathrm{L}} \mathrm{D}\left(\mathrm{C}_{\mathrm{m}, \mathrm{i}}\right) \cdot \mathrm{i}
\end{aligned}
$$

Where $\mathrm{D}\left(\mathrm{C}_{\mathrm{m}, \mathrm{i}}\right)$ represents the logical value whether the user's SINR is distributed in the segment $i(1 \leqslant i \leqslant \mathrm{~L})$. If the user's SINR is distributed in the segment $i, \mathrm{D}\left(\mathrm{C}_{\mathrm{m}, \mathrm{i}}\right)=1$, otherwise 0 . Here $\mathrm{D}_{\mathrm{j}}$ represents the USD of micro-cell $j$, which means the difference about channel quality conditions of the different users within the micro-cell. This provides an important reference to ensure user fairness.

Figure 2 shows the CDF curves of user's SINR in six micro-cells. The performance of the SINR for all users in the cell distribution reflects differences in the quality about user channel conditions.

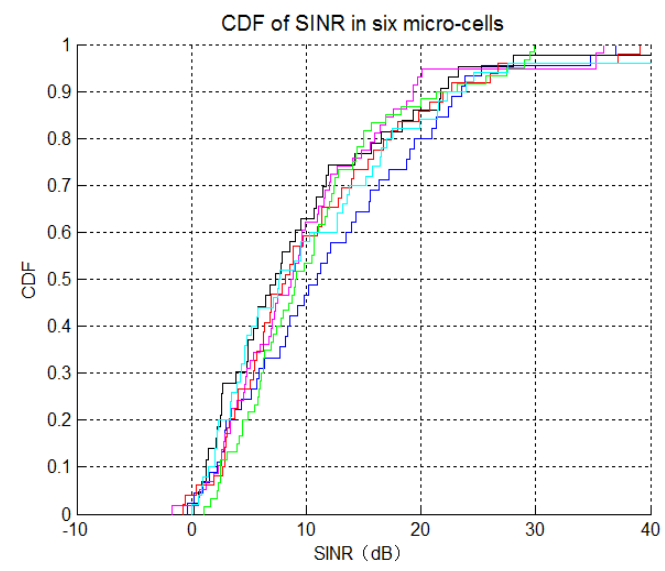

Figure 2. The CDF curve of user's SINR in six micro-cells

Case 1: The User SINR Dispersion of one micro-cell is low, which means that all the UE within the micro-cell have the similar channel conditions, so the user fairness parameter is a secondary consideration. At this point the system throughput can be improved greatly when we increase the number of resource blocks.

Case 2: The User SINR Dispersion of one micro-cell is high, which means that the users in the micro-cell have very 
different channel conditions, then the user fairness parameter value is the major consideration and the number of resource blocks should be reduced to ensure user fairness. Finally the system improves the SINR discrete case through resource scheduling, power control and other methods after obtain the feedback of differences in the channel conditions.

The SINR of the user represents the comprehensive situation of attenuation, interference and noise about user channel, determines a user's ability to receive resources. The USD indicates the relative condition of the user channel quality, as a main parameter of user fairness for resource allocation, can make more efficient use of system resources. We introduce the idea of the game theory in order to solve the equilibrium problem between system throughput and user fairness.

\section{GAMe Method}

Base on the analysis in section 2, the overall performance of the LTE-A relay system depends upon the maximization of system throughput and user fairness. The proposed game method plots the participators as normalized throughput and user fairness. The game target is to maximize the system throughput in the case of ensuring user fairness. The basic parameters to measure a cell's resource utilization are throughput and fairness. It's not correct to make a single pursuit of increasing throughput or fairness, it's reasonable to achieve the balance with an appropriate proportion of throughput and fairness.

\section{A. Balance degree for throughput and fairness}

This paper proposes the concept of cell balance degree, which means that the proportional relationship between normalized throughput and user fairness in the system. The adjustment of the cell balance degree directly affects the cell throughput and user fairness after the resource allocation.

The cell balance degree is defined as the quantization parameter ratio between the normalized throughput factor $\mathrm{R}$ and the user fairness factor $\mathrm{C}$. Cell balance degree as the adjustment of constraint parameters for the system throughput and user fairness, making the micro-cell of the higher throughput also requires a higher fairness. Similarly, the micro-cell with poor fairness requires sacrificing some throughput to ensure the fairness is not too low, and this part of the resources should be allocated to the micro-cell with a better fairness.

Based on the above analysis, the system performance depends on the resource allocation balance between the system throughput and user fairness. Unbalanced distribution will lead to a waste of radio resources even cause users' vicious competition of the resource. We only concern resource allocation and system performance of one single cell because we only consider the users and the radio resources within this district.

\section{B. System utility function of game}

The system performance can be represented by a utility function. We assume that there are $m$ UEs and $n$ RNs in the cell within the time T. Considering the relationship between cell throughput and user fairness, the utility function of the system is defined as follow.

$$
\begin{aligned}
& U\left(c_{i}, p_{j}, \theta\right)=\prod_{i=1}^{m} c_{i} \prod_{j=1}^{n}\left(p_{j}-P_{j}\right) \\
& \text { s.t } \quad \sum_{i=1}^{m} c_{i}=\theta G \\
& \text { s.t } \quad \sum_{j=1}^{n} p_{j}=(1-\theta) G
\end{aligned}
$$

Where $\mathrm{p}_{\mathrm{j}}$ represents the normalized actual rate of user $j$, $P_{j}$ represents the minimum required rate, $c_{i}$ represents the channel fairness degree of micro-cell $i$, which is the reciprocal of $D_{j}$. $\theta$ represents the proportional relation between the normalized throughput factor $\mathrm{R}$ and the user fairness factor $\mathrm{C}$, as the cell balance degree. $G$ represents the sum of normalized throughput and the user fairness.

Utility function consists of two parts, one part is the normalized throughput, and the other is user fairness. The utility function represents the obtained benefits when the normalized throughput and user fairness are in a balance proportion.

In this paper, the solution to the problem is maximizing the value of formula (4) under the conditions of equation (5) and equation (6). In this case, the exact value of function $U\left(c_{i}, p_{j}, \theta\right)$ is not considered. The parameter $\theta$ represents the best ratio between normalized throughput and user fairness of the optimal system performance when the function obtains the maximum value.

To simplify the calculations, we define $u(\theta)$ as follow.

$$
\mathrm{u}\left(\mathrm{c}_{\mathrm{i}}, \mathrm{p}_{\mathrm{j}}, \theta\right)=\ln \left(\mathrm{U}\left(\mathrm{c}_{\mathrm{i}}, \mathrm{p}_{\mathrm{j}}, \theta\right)\right)
$$

That's to say: 


$$
\mathrm{u}\left(\mathrm{c}_{\mathrm{i}}, \mathrm{p}_{\mathrm{j}}, \theta\right)=\sum_{\mathrm{i}=1}^{\mathrm{m}} \ln \mathrm{c}_{\mathrm{i}}+\sum_{\mathrm{j}=1}^{\mathrm{n}} \ln \left(\mathrm{p}_{\mathrm{j}}-\mathrm{P}_{\mathrm{j}}\right)
$$

Using Lagrange Multiplier Method to have the extreme value, we have the following equation.

$$
\begin{aligned}
& \mathrm{L}\left(\mathrm{c}_{\mathrm{i}}, \mathrm{p}_{\mathrm{j}}, \mathrm{P}_{\mathrm{j}}, \alpha, \beta\right)=\sum_{\mathrm{i}=1}^{\mathrm{m}} \ln \mathrm{c}_{\mathrm{i}}+\sum_{\mathrm{j}=1}^{\mathrm{n}} \ln \left(\mathrm{p}_{\mathrm{j}}-\mathrm{P}_{\mathrm{j}}\right)- \\
& \alpha\left(\sum_{\mathrm{i}=1}^{\mathrm{m}} \mathrm{c}_{\mathrm{i}}-\theta \mathrm{G}\right)-\beta\left(\sum_{\mathrm{j}=1}^{\mathrm{n}} \mathrm{p}_{\mathrm{j}}-(1-\theta) \mathrm{G}\right)
\end{aligned}
$$

To obtain the value of $\theta$ with the best performance of $u(\theta)$, we can find the partial differential of $c_{i}$ and $p_{j}$, and eliminate the unknown quantity $\alpha$ and $\beta$. The value of $\theta$ is as follow.

$$
\theta=\left(\frac{\sum_{j=1}^{n} P_{j}}{G \cdot n}+\frac{1}{m}\right) \cdot \frac{1}{\frac{1}{m}+\frac{1}{n}}
$$

From (10) and the former analysis, $\theta$ is between 0 and 1 , which means our radio resource allocation to normalized throughput and user fairness is rationality.

\section{The Balanced Aim algorithm}

According to the above, we propose a Balanced Aim (BA) allocation algorithm to achieve the optimal balance of system throughput and user fairness as the system goal. The BA allocation algorithm is mainly based on User SINR Dispersion of micro-cells which is influenced by the number of UE and $\mathrm{RN}$. The process of the algorithm is as follows:

1) Obtain the channel condition parameters and the distribution of UEs and RNs.

2) Compute the SINR of UEs and the access conditions for RNs and eNB.

3) Compute the User SINR Dispersion $D_{j}$ of micro-cell $j$.

4) Obtain the system parameter $c_{i}$ and $p_{j}$, generate system utility function $U\left(c_{i}, p_{j}, \theta\right)$.

5) Solving function $u(\theta)$ and obtain the cell balance degree $\theta$.

6) Allocate the resources according to the balance degree $\theta$.

It is depend on the balance degree $\theta$ that the number of resource blocks should be increased to improve system throughput or it should be reduced to ensure user fairness.

\section{Simulations}

In this section, we evaluate the effectiveness of our proposed BA allocation algorithm by means of simulations. These simulations were conducted with MATLAB. System-level simulation was performed on a network consisting of $1 \mathrm{eNB}$ with $6 \mathrm{RNs}$ in one cell. Main simulation parameters (path-loss, antenna, power) were taken from [14].
Figure 3 shows The User SINR Dispersion of 6 different micro-cells. It is obvious that the six micro-cells get great difference in The User SINR Dispersion although the users have an uniform random distribution, which means the Channel Quality of the six micro-cells is not fair.

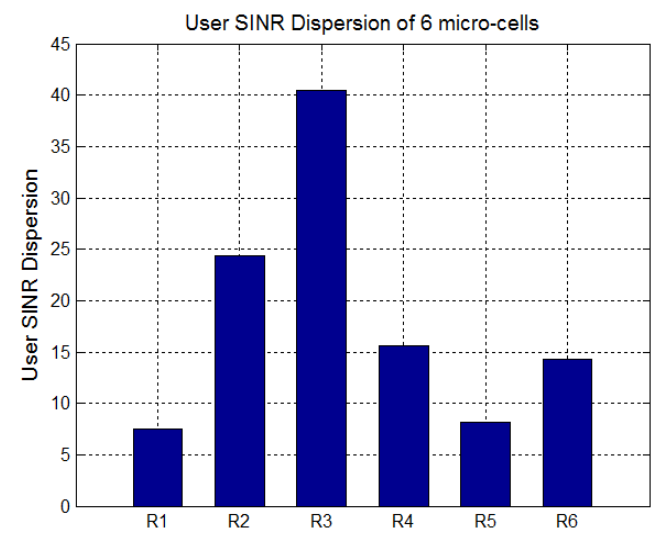

Figure 3. The User SINR Dispersion of 6 different micro-cells

The traditional scheduling algorithms include RR, Max $\mathrm{C} / \mathrm{I}$ and PF in the RRM of LTE-Advanced system. And the PF scheduling algorithm has better overall performance in terms of throughput and fairness. The simulation will simultaneously compare the proposed BA allocation algorithm with the $\mathrm{PF}$ scheduling algorithm and the PF scheduling algorithm with Relay (R_PF scheduling algorithm). Figure 4 shows the system throughput with 3 different scheduling algorithms and Figure 5 shows the user fairness. It is clear that the BA scheduling algorithm has the better performance in both system throughput and user fairness than others.

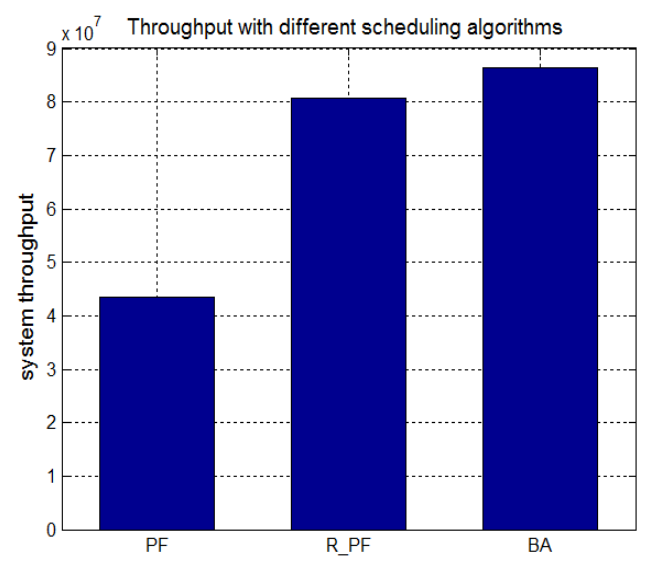

Figure 4. The system throughput with 3 different scheduling algorithms 


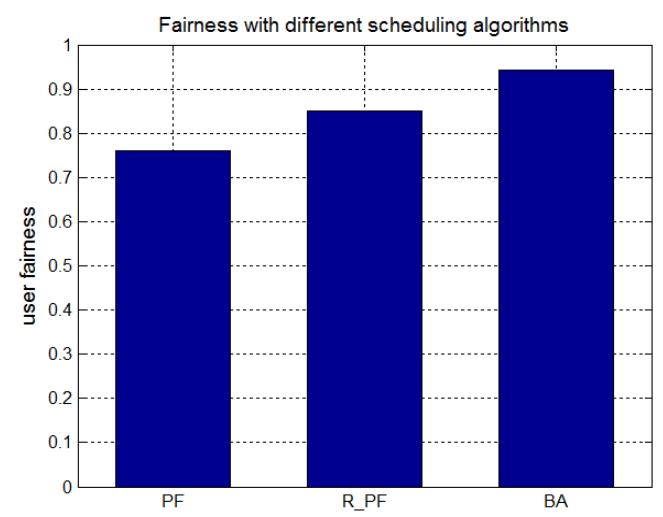

Figure 5. The user fairness with 3 different scheduling algorithms

Figure 6 and Figure 7 show the average throughput performance of above three scheduling algorithms (PF, PFr, BA) with different number of users. Although the BA scheduling algorithm didn't get the best average throughput performance when the user number is at 100 and 160 in Figure 6 , but it is obvious that the BA scheduling algorithm has a better performance basically when the number of users changes in Figure 7.

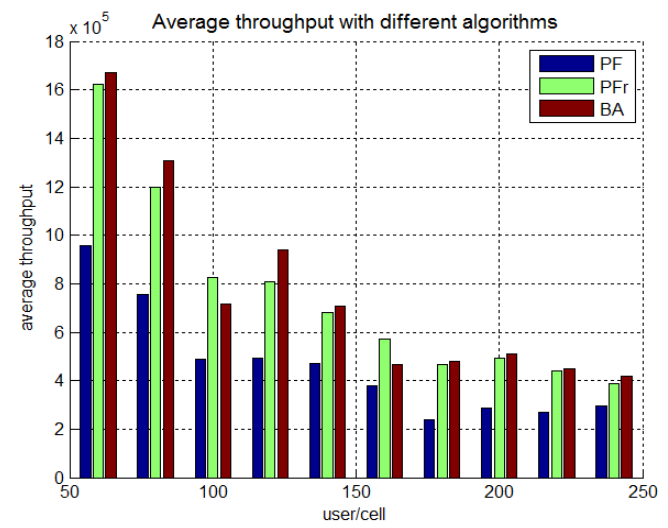

Figure 6. The average throughput with 3 different scheduling algorithms

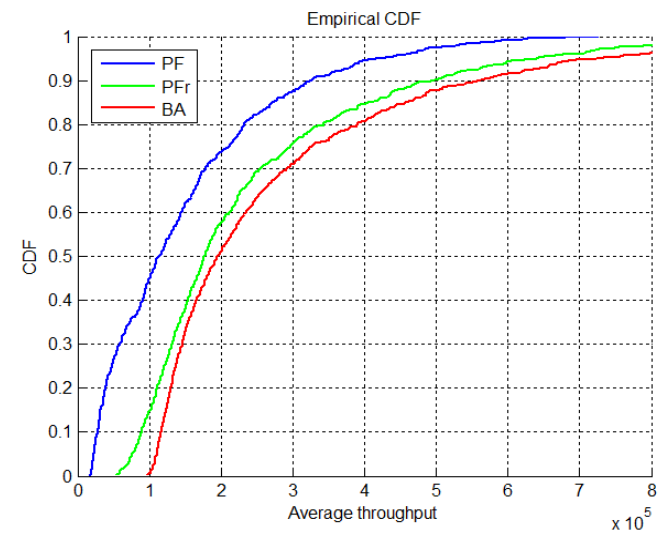

Figure 7. The CDF of average throughput with 3 different algorithms

\section{CONCLUSIONS}

LTE-A technology needs to meet different requirements with bandwidth and QoS of voice, data, video and other types of business. But the user experience on lots of business is limited because of the restrictive condition of radio resource. Radio resource management strategy will directly affect the quality of service of wireless networks. For balancing the performance between system throughput and user fairness of LTE-A system, we propose USD degree as the main parameter of resource allocation. Simulation results show that the BA scheduling algorithm which combined throughput, user fairness and game theory makes the system resource allocation more efficient, improves the overall performance of the system.

\section{ACKNOWLEDGMENT}

This work is supported by the National Natural Science Foundation of China (No.61071126) and the National Science and Technology Major Project Foundation "New Generation Broadband Wireless Mobile Communications Network" （No.2012ZX03001029-003、2012ZX03001008-003）.

\section{REFERENCES}

[1] Dan Chen, Hong Ji, and Leung V.C.M, "Distributed Best-Relay Selection for Improving TCP Performance Over Cognitive Radio Networks: A Cross-Layer Design Approach,” IEEE Journal on Selected Areas in Communications, vol. 30, pp. 315-322, February 2012.

[2] Alam M.S, Mark Jon W, and Xuemin Shen, "Relay Selection and Resource Allocation for Multi-user Cooperative LTE-A Uplink," 2012 IEEE International Conference on Communications (ICC), pp 5092-5096, 2012.

[3] Alam M.S, Mark Jon W, and Xuemin Shen, "Relay Selection and Resource Allocation for Multi-User Cooperative OFDMA Networks," IEEE Transactions on Wireless Communications, vol. 12, pp. 2193-2205, May 2013.

[4] Su Yi, and Ming Lei, "Backhaul Resource Allocation in LTE-Advanced Relaying Systems," 2012 IEEE Wireless Communications and Networking Conference: MAC and Cross-Layer Design, pp.1207-1211, 2012.

[5] Chun-I Kuo, Sau-Hsuan Wu, and Chun-Kai Tseng, "Robust Linea Beamformer Designs for Coordinated Multi-Point AF Relaying in Downlink Multi-Cell Networks," IEEE Transactions on Wireless Communications, vol. 11, pp. 3272-3283, September 2012. 
[6] Mauricio Iturralde, Anne Wei, Tara Ali Yahiya, and André-Luc Beylot, "Resource Allocation for Real Time Services Using Cooperative Game Theory and a Virtual Token Mechanism in LTE Networks," The 9th Annual IEEE Consumer Communications and Networking Conference, pp. 879-883, 2012.

[7] Qian Li, Rose Qingyang Hu, Yi Qian, and Geng Wu, "Intracell Cooperation and Resource Allocation in a Heterogeneous Network with Relays," IEEE Transactions on Vehicular Technology, vol. 62, pp. 1770-1784, 2013.

[8] M. Salem, A. Adinoyi, M. Rahman, H. Yanikomeroglu, D. Falconer, and Young-Doo Kim, "Fairness-aware radio resource management in downlink OFDMA cellular relay networks," IEEE Transaction on Wireless Communications, vol. 9, pp. 1628 - 1639, May 2010.

[9] M. Salem, A. Adinoyi, H. Yanikomeroglu, and D. Falconer, "Opportunities and challenges in OFDMA-based cellular relay networks: A radio resource management perspective," IEEE Transactions on Vehicular Technology, pp. 2496-2510, June 2010.

[10] M. Salem, A. Adinoyi, H. Yanikomeroglu, D. Falconer and Young-Doo Kim, "A Fair Radio Resource Allocation Scheme for Ubiquitous High-data-rate Coverage in OFDMA-based Cellular Relay Networks," IEEE Global Telecommunications Conference, pp. 1-6, 2009.

[11] W. Liping, J. Yusheng, and L. Fuqiang, "A Semi-distributed Resource Allocation Scheme for OFDMA Relay-Enhanced Downlink Systems," IEEE GLOBECOM Workshops, pp. 1-6, 2008
[12] M. Kaneko, P. Popovski, and K. Hayashi, "Throughput Guaranteed Resource Allocation Algorithms for Relay Aided Cellular OFDMA System," IEEE Transactions on Vehicular Technology, vol. 58, pp. 1951-1964, 2009.

[13] 3GPP TS36.211 v9.1.0, "Slot Structure and Physical Resource Elements," 2010.

[14] 3GPP TR 36.814 v1.4.1, "Further Advancements for E-UTRA Physical Layer Aspects," 2009.

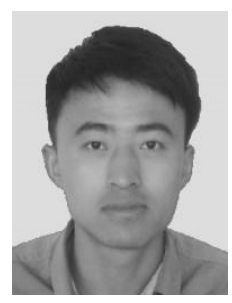

Jintao DU, was born in 1987. He is currently pursuing his M.S. degree in Broadband mobile Internet from Xi'an University of Post and Telecommunications. His current research interests include wireless broadband network, network management and control, radio resource management in LTE-A system.

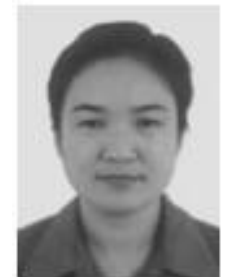

Jihong ZHAO, was born in 1963, Ph.D., professor of Xi'an Jiaotong University and Xi'an University of Posts and Telecommunications. Her current research interests include wireless broadband network, mobile Internet, network management and control. 